\title{
GPIM AF-M315E Propulsion System
}

\author{
Ronald A. Spores ${ }^{1}$, Robert Masse $^{2}$, Scott Kimbrel ${ }^{3}$ \\ Aerojet Rocketdyne, Redmond, WA, 98073 \\ Chris McLean ${ }^{4}$ \\ Ball Aerospace and Technologies Corporation, Boulder, Co, 80301
}

\begin{abstract}
The NASA Space Technology mission Directorate's (STMD) Green Propellant Infusion Mission (GPIM) Technology Demonstration Mission (TDM) will demonstrate an operational AF-M315E green propellant propulsion system. Aerojet-Rocketdyne is responsible for the development of the propulsion system payload. This paper statuses the propulsion system module development, including thruster design and system design; Initial test results for the $1 \mathrm{~N}$ engineering model thruster are presented.
\end{abstract}

The culmination of this program will be high-performance, green AF-M315E propulsion system technology at TRL 7+, with components demonstrated to TRL 9, ready for direct infusion to a wide range of applications for the space user community.

\begin{tabular}{|c|c|}
\hline & Nomenclature \\
\hline$E M$ & $=$ Engineering model \\
\hline ESPA & $=$ EELV secondary payload adapter \\
\hline GPIM & $=$ Green Propellant Infusion Mission \\
\hline HAN & $=$ Hydroxyl ammonium nitrate \\
\hline & $=$ Specific Impulse \\
\hline IHPRPT & $=$ Integrated High Payoff Rocket Propulsion Technology \\
\hline SCAPE & $=$ Self-Contained Atmospheric Protection Ensemble \\
\hline TRL & $=$ Technology Readiness Level \\
\hline
\end{tabular}

\section{Introduction}

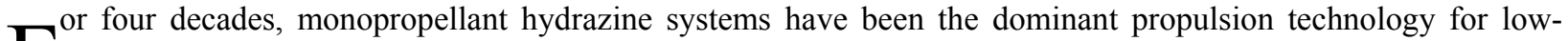
F total-impulse applications; however, expensive storage, handling, and disposal procedures are required to address the propellant toxicity and flammability hazards, which, though well established, continue to hinder efforts to reduce mission integration costs and schedule. While traditional green alternatives such as cold gas and electric propulsion may reduce schedule and cost impacts, their limited specific impulse and thrust respectively preclude their application to missions requiring high total impulse and/or thrust. As such, the last decade has seen a growing awareness that the development of a low-toxicity alternative offering performance better than hydrazine would yield substantial crosscutting benefits to NASA and all space users. Toward this objective, the NASA Space Technology Mission Directorate (STMD) has initiated the Green Propellant Infusion Mission (GPIM) program with the objective of completing the first on-orbit demonstration of a complete AF-M315E high-performance $(+50 \%$ density $-I_{\mathrm{sp}}$ compared to traditional hydrazine) green propellant propulsion system by the end of 2015 . Hosted on a Ball Aerospace BCP-100 ESPA-class spacecraft bus, the GPIM Technology Demonstration Mission (TDM) will employ an Aerojet-developed advanced monopropellant payload module as the sole means of on-board propulsion, performing a comprehensive battery of performance characterization and capabilities assessment maneuvers using both $1 \mathrm{~N}$ and $22 \mathrm{~N}$ thrusters ${ }^{1,2,3,4,5}$. The $1 \mathrm{~N}$ and $22 \mathrm{~N}$ thrust classes represent the largest segments of the monopropellant thruster market. the specific intent of the GPIM program is to advance AF-M315E technology to a readiness level suitable for immediate infusion in both short-duration and extended near-future applications. The

\footnotetext{
${ }^{1}$ Manager of Programs, Advanced Development, Aerojet-Rocketdyne, Redmond, WA.

${ }^{2}$ Chief Engineer, Advanced Development, Aerojet-Rocketdyne, Redmond, WA.

${ }^{3}$ Propulsion Systems Lead, In-space Systems, Aerojet-Rocketdyne, Redmond, WA.

${ }^{4}$ Principal Investigator GPIM, Mission Systems, Ball Aerospace and Technologies Corp., Boulder, Co.
} 
propulsion system under development incorporates principally heritage hydrazine system components selected for the long-duration compatibility of their materials of construction with the new propellant.

Aerojet Rocketdyne's commitment to green propulsion has spanned two decades and a wide range of propellant options. Initial experience was gained with HAN/glycine and HAN/methanol formulations ${ }^{6}$. Shifting focus to AFRL-developed AF-M315E ionic liquid advanced monopropellant in 2001, Aerojet Rocketdyne's green thruster technologies had matured to TRL5 by 2011, meeting the IHPRPT Phase II objective of 50\% increased density-Isp over conventional hydrazine equivalents. Unique among a number of hydrazine alternatives that have emerged in recent years, AF-M315E is sufficiently green to enable safe handling in open containers for unlimited durations, whereas the properties and/or handling hazards (such as super-atmospheric vapor pressure or necessary stabilizers which may evaporate) of other current low-toxicity candidates preclude this. The summation of numerous development efforts and programs over many years, 2011 saw the first successful demonstration of more than 11.5 hrs firing life by an AF-M315E thruster employing a breakthrough patent-pending high-temperature catalyst-, heralding readiness for infusion into a wide range of NASA, DoD, and commercial missions.

\section{Payoff to NASA, Commercial and DoD Missions}

NASA science missions place a premium on performance, cost, robustness, and thermal requirements, all of which are enhanced by the use of GPIM's AF-M315E propulsion technology. AF-M315E offers higher performance than hydrazine, yields $12 \%$ higher $\mathrm{I}_{\mathrm{sp}}(257 \mathrm{vs} .235 \mathrm{sec})$, and is $45 \%$ more dense $(1.47 \mathrm{vs} .1 .00 \mathrm{~g} / \mathrm{cc})$, affecting both reduced propellant and tank mass. In addition to reduced test and loading costs owed to its low toxicity, AF-M315E simplifies the safe design and development of propulsion systems compared to hydrazine. Since leakage of AF-M315E is rated as a critical rather than catastrophic failure, only single-fault-tolerance is required for safety in handling flight systems. This alone accounts for significant savings, as redundant components are eliminated, yielding simpler architectures. Further, simpler and much less expensive design and verification criteria govern flight-qualification of fracture-critical hardware (e.g., propellant tanks) for non-hazardous propellants such as AF-M315E compared to hydrazine. .

With its lower minimum temperature threshold, AF-M315E yields an additional advantage of mitigating operational concerns related to long-duration system thermal management. Whereas hydrazine space tanks and lines must be heated at all times to prevent freezing, AF-M315E cannot freeze (it has a glass transition). During long coast periods an AF-M315E propulsion system may be allowed to fall to very low temperatures and later reheated for operation without risk of line rupture by phase-change-induced expansion. This can be particularly beneficial to planetary spacecraft and planetary ascent vehicles, which can call for years of propellant storage in cold environments. Tests also have demonstrated AF-M315E to has a significantly reduced sensitivity to adiabatic compression than hydrazine.

AF-M315E also offers comparable performance (density $-\mathrm{I}_{\mathrm{sp}}$ ) to traditional storable bipropellants for low $\Delta \mathrm{V}$ missions while employing roughly half the number of components, thereby retaining the well-established increased reliability and reduced cost of traditional monopropellants. Many design issues and failure modes associated with long-duration interplanetary missions (e.g. control of mixture ratio, of propellant vapor diffusion and reaction, oxidizer flow decay) do not apply to an equally capable AF-M315E system.

The cost savings of green propellants associated with simplified range operations are quantifiable. The average contractual cost to load a NASA mission with conventional propellants is $\$ 135,000^{\text {Error! Bookmark not defined. The cost }}$ for loading with AF-M315E will be a small fraction of this, and the associated schedule significantly expedited. Per current conventions, propellant loading operations require one shift for setup in SCAPE, a second shift waiting for propellant test confirmations, a third shift or more for actual loading, and a final additional shift to break down the setup, during which all remaining launch processing staff must wait at costs exceeding $\$ 100 \mathrm{k} /$ day for a typical Class B NASA mission. An early Aerojet Rocketdyne study evaluating replacement of hydrazine with a HANbased advanced monopropellant for Centaur RCS on an Atlas launch vehicle concluded ground support costs of fueling could be reduced by two-thirds ${ }^{7}$.

\section{GPIM Propulsion System}

Under development as a self-contained module to allow independent assembly at Aerojet Rocketdyne for subsequent integration into the bus, the GPIM demonstration payload, illustrated in Figure 2 and shown in schematic in Figure 1, will deliver 50\% more impulse than a comparably-packaged hydrazine system. Designed to attach to the Ball Aerospace BCP-100 bus via its standard payload interface plate (PIP), the GPIM demonstration payload 
comprises a simple, single-string, blow-down AF-M315E advanced green monopropellant propulsion system employing four $1 \mathrm{~N}$ attitude-control thrusters and a single $22 \mathrm{~N}$ primary divert thruster. The propellant feed manifold's principal components, consisting of a standard diaphragm propellant tank, latch valve, and service valves, represent all flight-proven (TRL 9 with hydrazine propellant) designs selected specifically for the long-term compatibility of their materials of construction with AF-M315E. Redundant pressure transducers monitor gas-side propellant tank pressure (and hence propellant consumption). Thrusters are mounted on the upper deck of a box-like payload primary structure. The $22 \mathrm{~N}$ primary divert thruster is mounted on the spacecraft centerline with the thrust axis pointed through the PIP-mounted propellant tank and spacecraft centers of mass. The four $1 \mathrm{~N}$ thrusters are canted on brackets at the corners of the upper deck to maximize the moment arm to the spacecraft center of mass, and thereby control authority and resolution of impulse measurement by the bus attitude and orbit determination and control (AODC) sensors. The remaining propulsion system components are consolidated on a component panel attached to the underside of the upper deck, except for the two service valves, which mount to a separate bracket positioned for easy access during fueling and range operations, and two pressure transducers which are mounted to the PIP deck.

Design considerations for the AF-M315E propulsion system are mostly similar to a traditional hydrazine system, with a few special considerations. Principally, all system components must be compatible with AF-M315E especially for longer duration missions. As the general schematic layout is identical to single string blow-down hydrazine systems commonly employed on small spacecraft, many of the same general design guidelines apply. The AF-M315E system however, is far less hazardous than a traditional hydrazine system when considering range safety requirements. The propellant is far less prone to leakage (due to higher viscosity), is non-toxic if leaked, and the thrusters cannot inadvertently fire without having first preheated catalyst beds. KSC range safety personnel have assigned AF-M315E a reduced hazard severity classification of "critical" per MIL-STD-882E (Standard Practice for System Safety). In contrast, hydrazine external leakage is ranked a "catastrophic" hazard rating. Per Range Safety AFSPCMAN 91-710 requirements, a classification of "critical" or less only requires a two-seal inhibits to external leakage; hence no additional latch valves other isolation device are required in the feed system despite the fact that the advanced monopropellant thrusters employ only single-seat valves. This approach reduces the complexity, power, and mass of the thruster valve, while simplifying electrical interfaces, all without sacrificing mission reliability.

Other differentiating design considerations arise principally from differences in the thermal characteristics of AF-M315E vs. conventional thrusters. Due to the advanced monopropellant thrusters' elevated minimum start temperature, catalyst bed preheat power requirements are higher compared to a conventional hydrazine system. This increase is partially offset, however, by the reduced power needs of the thrusters' single seat valves, as well as much lower power required for system thermal management during non-operating periods enabled by the propellant's demonstrated storage stability very low temperatures (although current CONOPS for the GPIM mission call for the propellant to be maintained within nominal system operating range). Radiation and conduction from the advanced

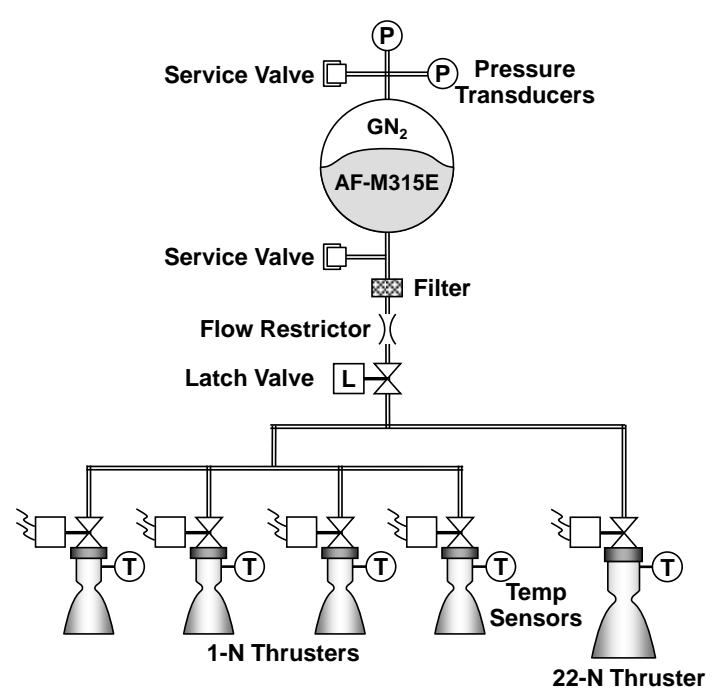

Figure 2 Propulsion System Schematic

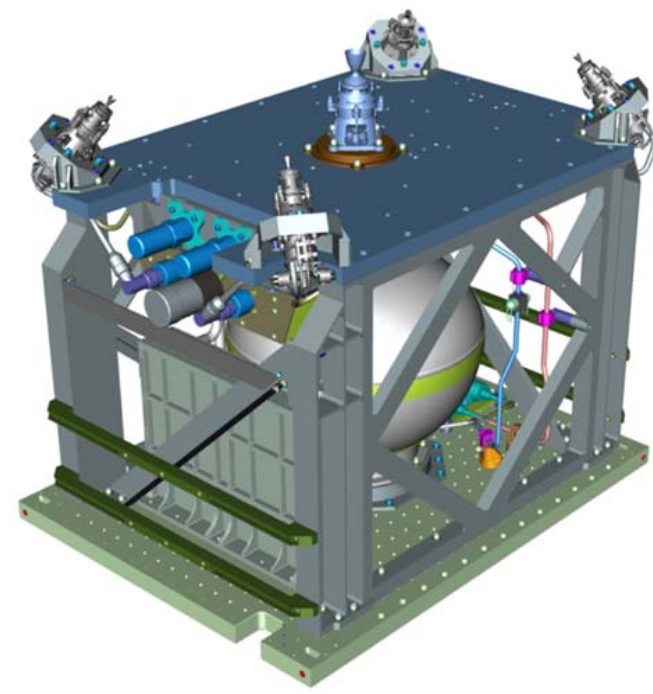

Figure 1 AF-M315E Propulsion System

3

American Institute of Aeronautics and Astronautics 
monopropellant thrusters' high temperature chambers also impart a moderate increase in the thermal load to the system mounting interface.

\section{AF-M315E Green Advanced Monopropellant Thrusters}

The Aerojet Rocketdyne 1N (GR-1) and 22N (GR-22) advanced monopropellant thrusters to be employed on GPIM represent the culmination of over two decades of research, spanning the development of enabling hightemperature test and data acquisition techniques applied to testing of a number of candidate propellants, extensive evaluation and test of numerous material systems for structural components and catalysts, and thruster performance characterization ranging from less than one up to $670 \mathrm{~N}$ (150 lbf) thrust in both sea-level and vacuum environments. Throughout a large portion of over two decades of research, inherently high reaction temperatures associated with ionic liquid propellants, coupled with poorly understood ionic-liquid thruster stability dynamics, constrained both thruster life and operational duty cycle capabilities. The last several years, however, have yielded significant breakthroughs related to both materials and a fundamental understanding of the governing mechanics of ionic liquid thrusters necessary to design and fabricate robust, practical (duty-cycle-unlimited) thrusters with sufficient life capability to meet real mission needs. A key contributor to the rapid acceleration in maturation of AF-M315E thruster technology seen in recent times has been the advent of Aerojet's patent-pending LCH-240 high-temperature long-life catalyst, demonstrating sufficient endurance within the propellant's decomposition/combustion environment to extend thruster life over $15 \times$ compared to the prior state-of-the-art.

The GR-1 and GR-22 advanced monopropellant thrusters implement a common design strategy whereby the use of refractory alloys (to accommodate the flame temperature of the AF-M315E propellant) is confined to the thrust chamber, nozzle and an upper thermal isolation structure, such that much of the thruster can be fabricated with conventional alloys in common use on hydrazine thrusters today. Trade studies indicate this hybrid approach yields significant respective cost and power savings compared to evaluated alternatives entailing either all-refractory or bulkier, heavily-insulated conventional alloy construction. The resulting flight thruster designs, shown side-by-side for comparison in Figure 3, comprise a series-assembled valve, injector, catalyst-containing chamber, and nozzle bearing general resemblance to conventional catalytic hydrazine thrusters of corresponding thrust classes, with two readily notable differences.
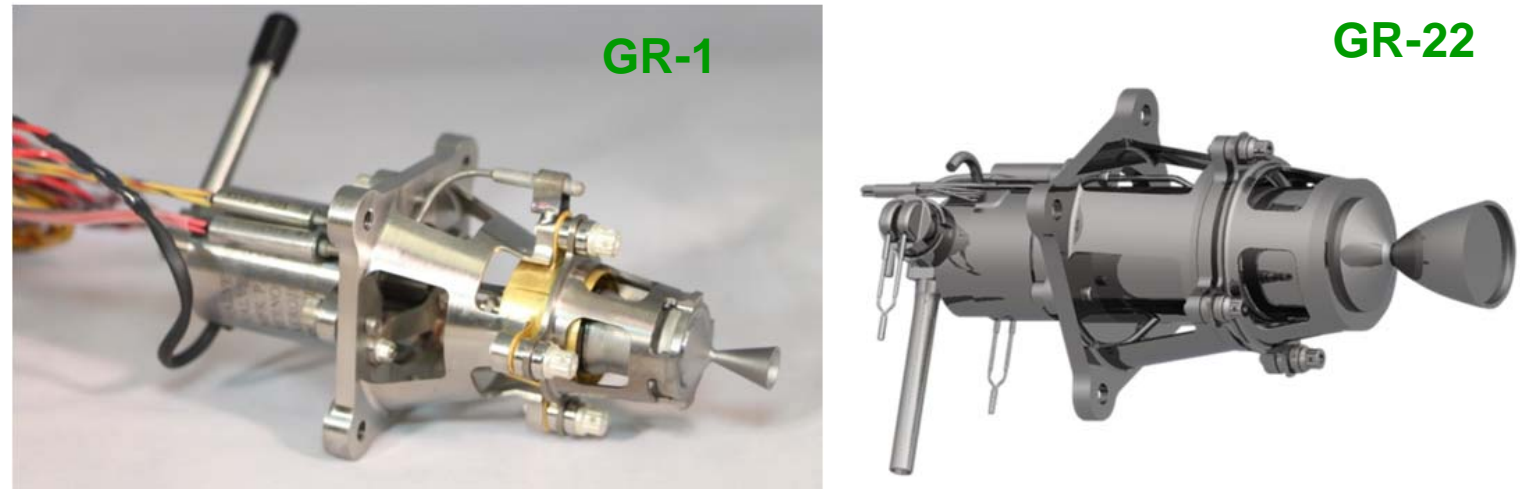

Figure 3 Aerojet GR 1 and GR 22 Thrusters

Most immediately apparent are the extended two-piece stand-off structures employed by both designs. These provide additional thermal isolation serving the dual roles of preventing overheating of the spacecraft interface by heat soak-back from the chambers during and following extended thruster firings, as well as limiting heat loss from the catalyst bed during thruster preheating, thereby minimizing power necessary to preheat the catalyst bed to the nominal start temperature. The GR-1 and GR-22 will be readily infusible into most applications likely to employ conventional monopropellants.

The GR-1 and GR-22 thrusters also employ notably smaller, single-seat valves with higher net reliability than the two-seat scheme generally favored for comparable hydrazine thrusters. This results from an inadvertent benefit inherent to specific properties of the ionic liquid propellant. Being more viscous than hydrazine, AF-M315E is intrinsically far less prone to leakage, such that the doubled risk of a thruster becoming inoperable in the event of either of two valve stages becoming inoperable is not justified. Moreover, having essentially no vapor pressure, AF-M315E will not self-pressurize or evaporate through small fissures such as a flaw in a valve seat, such that, in

4

American Institute of Aeronautics and Astronautics 
the very unlikely event that thruster valve leakage should occur, isolation of the downstream feed system by closing the upstream system latch valve would fully prevent any loss of propellant. Likewise for launch range operations, the innate safety of the propellant, accounting for its low vapor toxicity, and inability to activate un-preheated thrusters or react with external system and immediate work environment materials (unlike hydrazine), obviates the conventional rationale for the use of dual seat thruster valves. Thus, single seat valves provide higher mission assurance at lower mass, power (partially offsetting added preheat power requirements), and cost solution for the GPIM and future missions. Further, the added compactness of the GR-1 and GR-22 designs realized through the selection of single-seat valves has proven substantially facilitating in the close packaging of the GPIM demonstration system module, portending similar benefits to future ESPA-class spacecraft. Note that single seat valves have been used on many hydrazine-propelled spacecraft, and particularly prior NASA missions such as Cassini, Deep Impact, New Horizons, and Voyager (still successfully operating since its launch in 1977).

With a view to maximizing immediate infusability of the technology into both short-duration and extended missions, AF-M315E-specific material compatibility requirements (which differ from hydrazine) have been addressed in the selection of control valves for the GR-1 and GR-22 thrusters. In particular, as a mild acid, AF-M315E demonstrates long-term compatibility with a limited set of metals, none of which are ferromagnetic. Thus, the GR-1 and GR-22 thrusters employ largely new valve designs incorporating AF-M315E compatible wetted surfaces. The valves still derive considerable design and manufacturing process heritage from flight-proven products. Indeed, the GR-1 and GR-22 valve designs leverage existing process capabilities developed specifically for other applications necessitating isolation of valve ferromagnetics from working fluids.

The ongoing GPIM flight thruster development effort is structured in three overlapping phases. The first phase was executed last year (July 2013) sea-level testing of heavyweight hardware. This testing performed duty cycle mapping of (principally the 22-N) thruster over a comprehensive range to verify broad functional stability, thereafter to anchor thruster life models as operated at duty cycles and simulated feed pressure blow-down ratio closely approximating projected mission performance requirements. Guided by these test results, Engineering Models (EM) of both the $1 \mathrm{~N}$ and $22 \mathrm{~N}$ thrusters are being fabricated and extensively hot-fire tested in Phase 2. In Phase 3, flight designs will be finalized and flight (one each) units fabricated. All thrusters will undergo standardized acceptance testing, comprising shock, vibration, and a check-out hot-fire. On orbit, the thrusters will perform a series of maneuvers designed to both fully characterize thrust, Ibit, specific impulse, and thermal performance over a variety of duty cycles intended to encompass the full needs of near-future space applications.

\section{Thruster Performance}

Designed as functional alternatives to Aerojet Rocketdyne's $1 \mathrm{~N}$ class MR-103G and 22N class MR-106L, thrust vs. feed pressure characteristics for the GR-1 and GR-22 are presented in Figure 4, with key operating metrics summarized in Table 1.
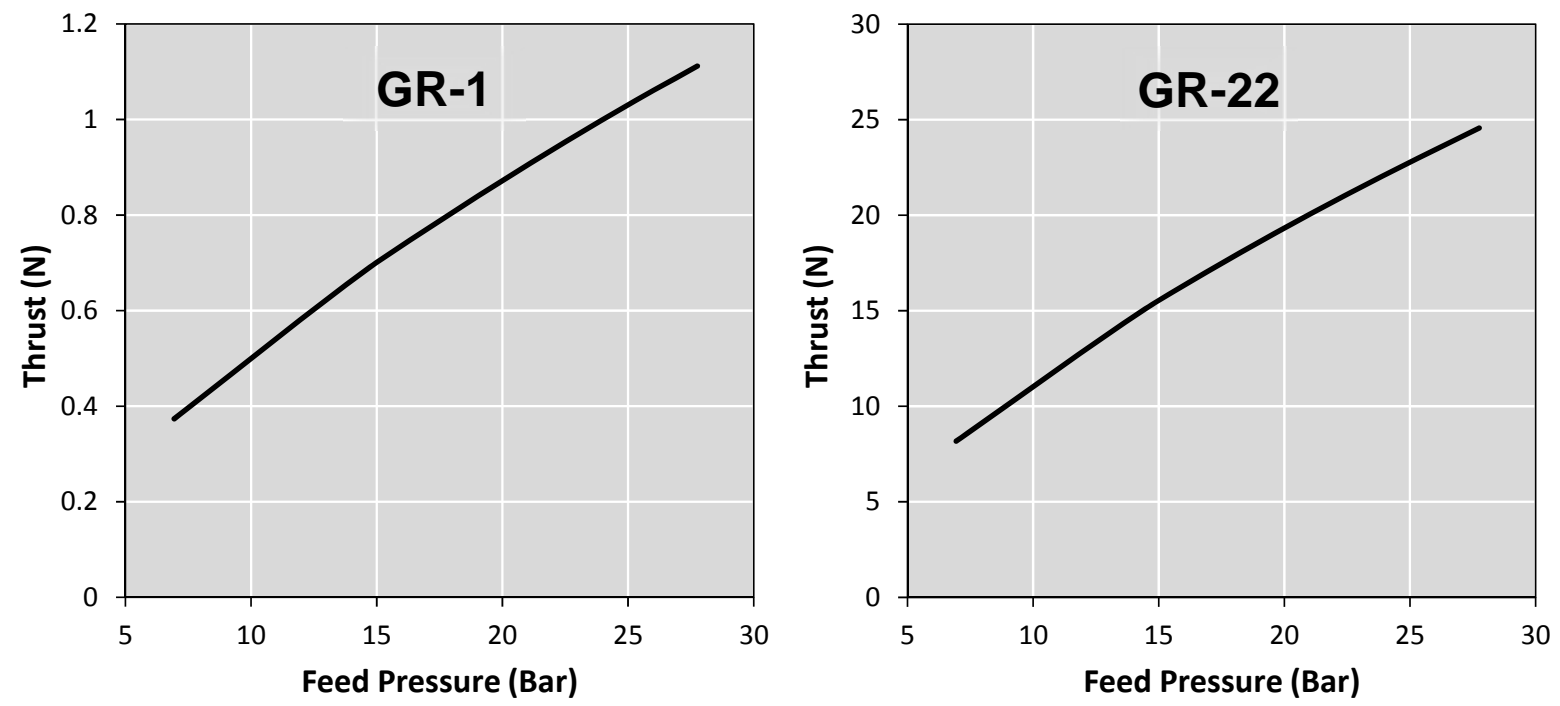

Figure 4 Aerojet Rocketdyne GR-1 and GR-22 Thrust vs. Feed Pressure Curves 
Table 1 Thruster Predicted Performance Summary

\begin{tabular}{|lrr|}
\multicolumn{1}{c}{} & \multicolumn{1}{c}{ GR-1 } & \multicolumn{1}{c|}{ GR-22 } \\
\hline Thrust (N) & $0.4-1.1$ & $8-25$ \\
\hline Feed Pressure (bar) & $6.8-27.6$ & $6.8-27.6$ \\
Nozzle Expansion Ratio & $100: 1$ & $50: 1$ \\
Valve Power (W) & 12 & 28 \\
Preheat Power (W) & 10 & 30 \\
Specific Impulse (s) & 235 & 250 \\
Total Impulse (N-s) & 23,000 & 74,000 \\
Minimum Impulse Bit (mN-s) & 8.0 & 116 \\
\hline
\end{tabular}

\section{Engineering Model Thruster Test Status}

Testing of the GPIM engineering model GR-1 thruster began on June 5, 2014 in Aerojet Rocketdyne's Advanced Propellants Test Lab (APTL, see Figure 5) with an extensive non-fire characterization of its vacuum preheat characteristics. Established as a significant expansion of its Redmond, Washington, USA-based advanced propellant development and testing capabililities, to date, this facility houses a single state-of-the-art $1.8 \mathrm{~m}$ dia $\times$ $2.6 \mathrm{~m}$ long cylindrical stainless steel high-altitude cell equipped with a 64-channel Dewetron $204 \mathrm{kS} / \mathrm{s}$ data acquisition system and dual Stokes 1739 combination vacuum pumps supporting testing down to 2.7 mbar. Thereafter, between the $10^{\text {th }}$ and $17^{\text {th }}$ of the same month, the thruster completed a series of pulse-mode and steadystate hot-fire acceptance tests (see Figure 6). As the refractory construction of the thrust chamber precludes direct chamber pressure monitoring, thruster performance and health were monitored via a fast-response thrust stand and a pitot probe directed into the plume at the nozzle exit plane, as well as a broad array of surface-mounted thermocouples. Upon completion of acceptance hot-fire testing, the engineering model underwent to protoflight vibration testing. All tests met or exceeded objectives. As such, the GR-1 engineering model thruster will shortly return to the APTL for an extended proto-flight life testing, to continue as required to determine operational end-oflife as defined by a maximum allowable roughness (determined via the pitot probe) of $\pm 50 \%$. 


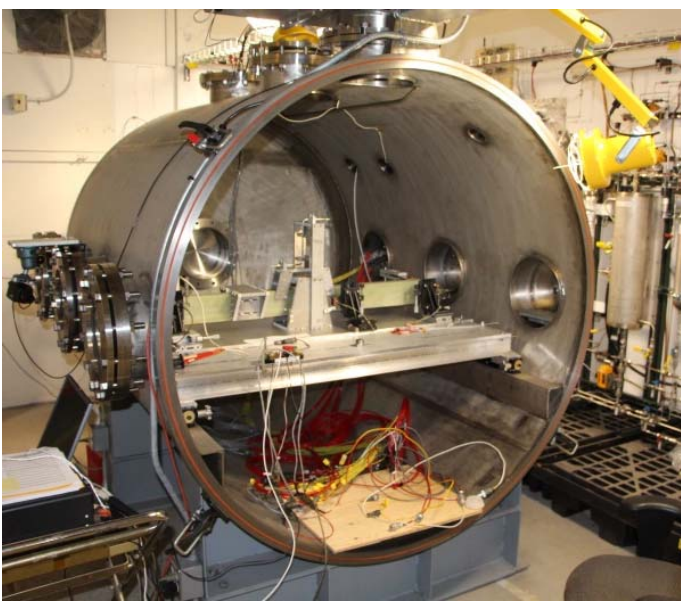

Figure 5 Aerojet Rocketdyne’s Advanced Propellants Test Lab

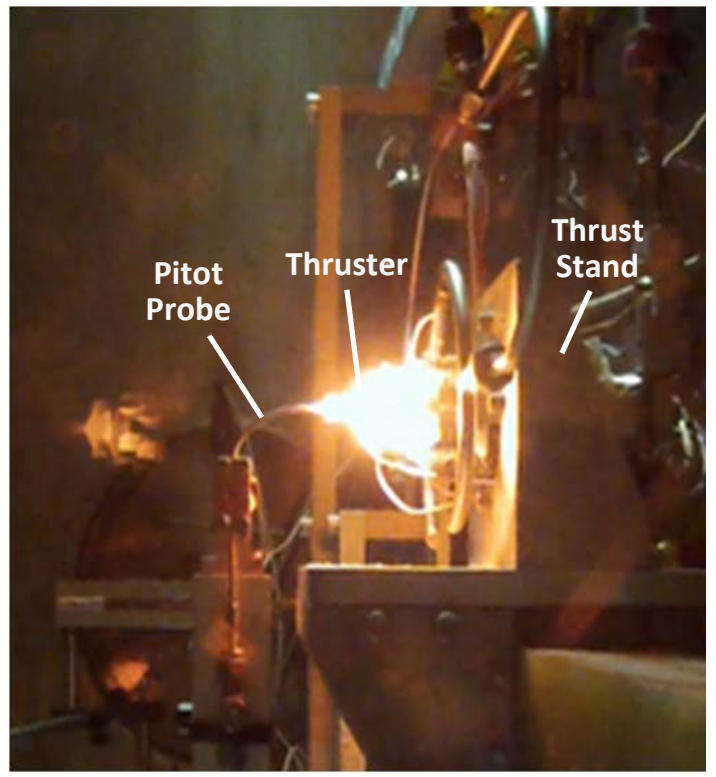

Figure 6 GR-1 Thruster in Operation

\section{Thruster Preheat Thermal Characterization}

Because the GPIM AF-315E thruster must reach a catalyst bed preheat temperature exceeding $285^{\circ} \mathrm{C}$ to be ready for general operation (it is possible to pulse start from lower temperatures), characterization and verification of the thermal models in non-firing modes of operation is critical to the success of the flight thruster designs. In Figure 7 selected thermocouple data, obtained through extended monitoring of thruster self-preheating under vacuum within the test cell, is compiled alongside baseline predictions by a thermal model employing representative boundary conditions. The data confirm consistently that sufficient margins, largely the result of conservatively assigned emissivity and contact resistance coefficients, were carried in the thruster thermal design to ensure robust margins for on-orbit operation. Resulting improvements to model fidelity will be employed to evaluate potential reductions in thruster preheat power as the GPIM program moves toward final design and fabrication of the flight demonstration thruster shipset.

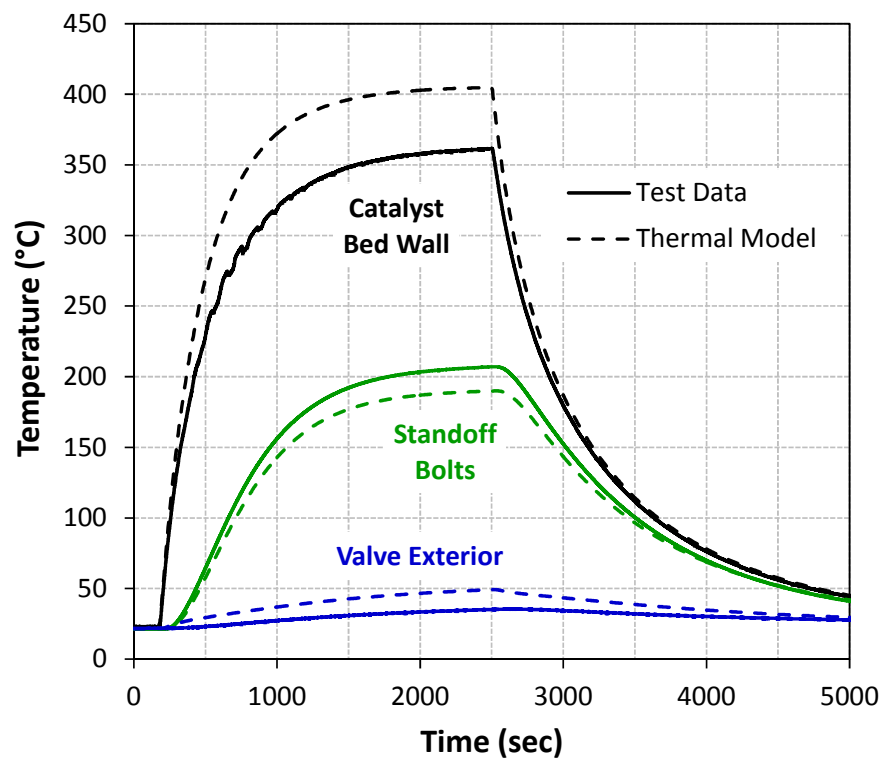

Figure 7 Predicted vs. Measured Thruster Warm-up Transients 


\section{Thruster Hot-Fire Acceptance Preliminary Test Results}

Figure 8 depicts thrust data from a series of steady burns operated from cold start over a range of tested feed pressures $\left(\mathrm{P}_{\mathrm{f}}\right)$. In each case, thrust demonstrates an expected rapid approach to a momentary plateau (at approximately $75 \%$ of nominal) before asymptoting to steady-state operation, a fundamental cold start thermal characteristic of porous bed reactors common to conventional hydrazine thrusters. Note that the apparent ringing near the beginning and end of each sequence represents an artifact of the thrust stand data reduction algorithm, and should not be interpreted as an actual performance characteristic. Measured steady state thrust vs. feed pressure is compared with design-level predictions in Figure 9. While the thruster does not fully reach maximum operating temperature within the tested 30-sec run duration, comparison of previous 1-N heavyweight thruster data to present measured mass flow rate and thrust yields an estimated maximum steady-state Isp approximating the predicted $235 \mathrm{sec}$. Throughout acceptance testing, roughness remained minimal and showed no signs of increase.

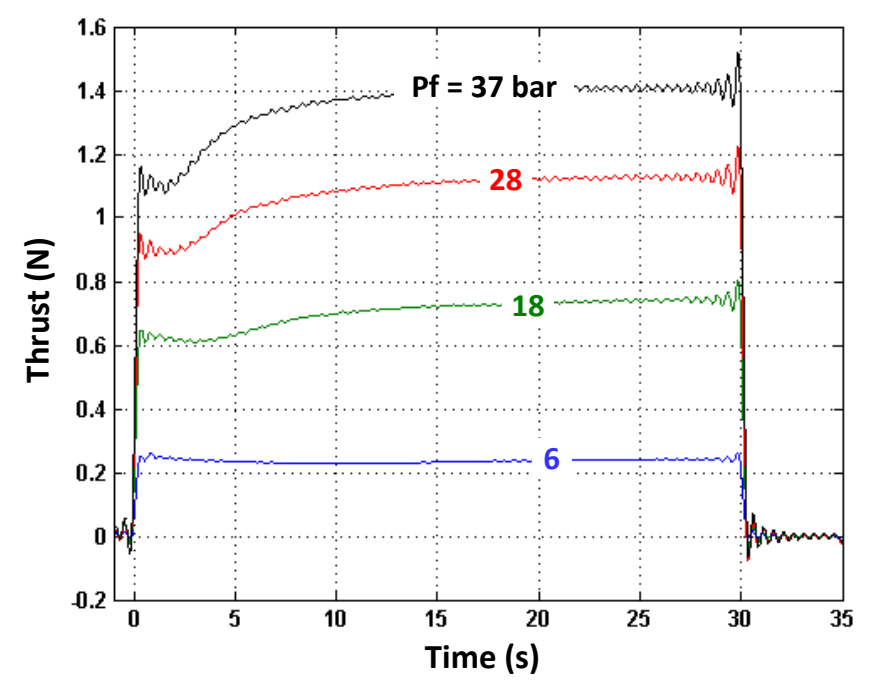

Figure 8 Thrust Traces for 30-sec Burns Operated from Cold Start Condition at Miscellaneous Feed Pressures

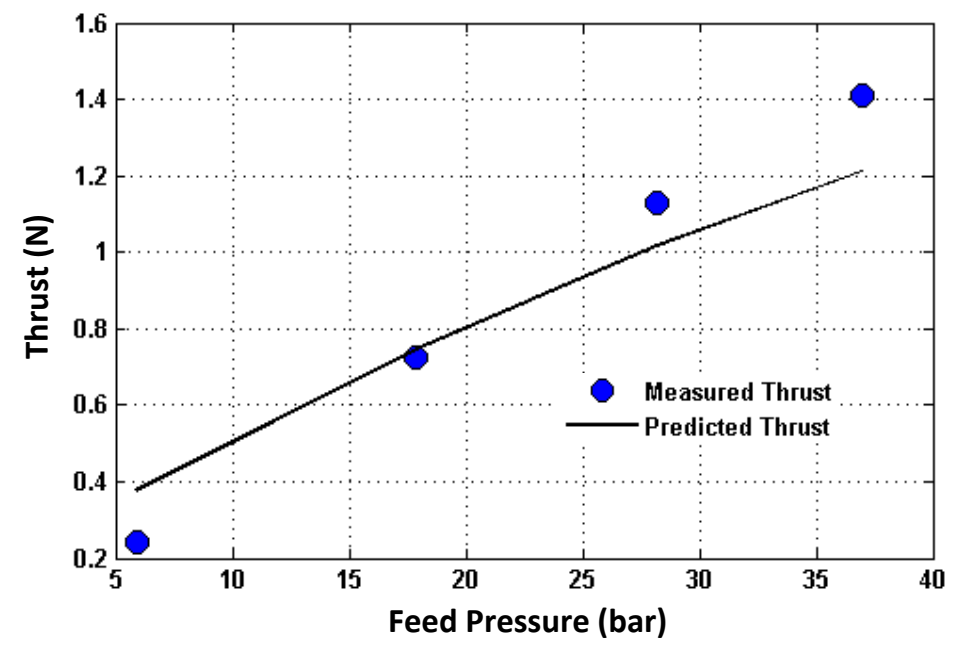

Figure 9 Measured vs. Predicted Thrust as a Function of Feed Pressure

Normalized start-up transient thrust profiles for the engineering model GR-1 (per direct measurement) vs. its heavyweight antecedent (as computed from thrust chamber measurements) are superimposed for comparison in Figure 10. The illustrated high degree of demonstrate similarity between the two test units confirms that the insulation scheme employed in the heavyweight unit provides sufficient thermal isolation of the thrust chamber such 
that heat dissipation to the walls is minimal, indicating all performance metrics previously established by heavyweight testing can be expected to carry forward for flight thrusters.

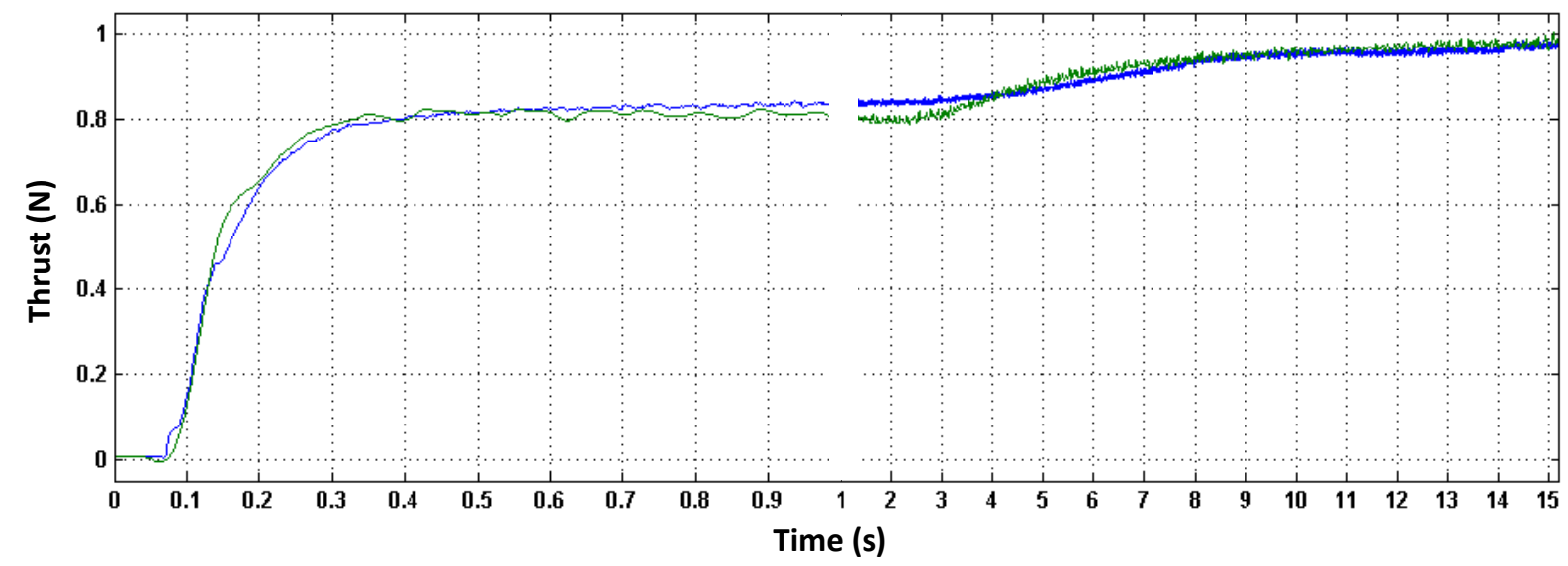

Figure 10 Normalized Comparison of Start-up Thrust Profiles for GR-1 Thruster vs. heavyweight 1-N Test Unit

Extensive pulse-mode performance characterization, to be conducted as part of the upcoming protoflight life test, is not yet complete. However, the executed hot-fire acceptance test plan did include a number of pulse-mode stability mapping sequence sufficient to verify the intended duty-cycle independent operability of the design. Examples of two pulse trains, executed from the nominal $\sim 315^{\circ} \mathrm{C}$ preheat condition varying in commanded on-time by an order of magnitude, are shown in Figure 11 . Whereas the $200 / 178 \mathrm{msec}$ on/off sequence is observed to vary continuously over twenty executed pulses, the $20 / 180 \mathrm{msec}$ on/off sequence, corresponding to a lower terminal mean catalyst bed temperature, reaches cyclic thermal equilibrium relatively quickly, demonstrating at near-limitcycle pulse-to-pulse impulse bit variability of $<0.6 \%$ root-mean-square-standard deviation. This is significantly less than the variability typical of $1-\mathrm{N}$ class hydrazine thrusters operating at similar duty cycles.

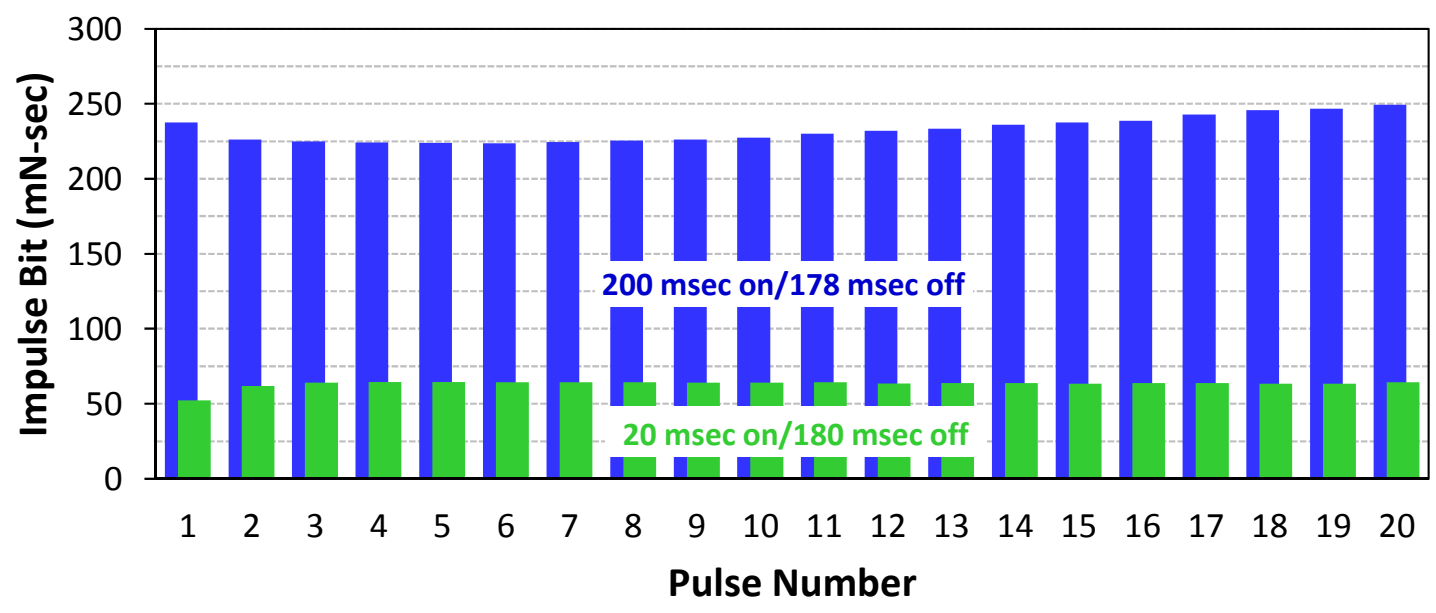

Figure 11 Ibit for Select Hot-fire Acceptance Test Pulse-Mode Sequences

\section{System Components Maturation Status}

AF-M315E propellant is mildly acidic which can result in a small amount of leaching of some common aerospace materials with long term propellant exposure. In addition, this fuel can act as both a reducing agent or as an oxidizing agent, so establishing metal passivation is more difficult than for pure reducing (hydrazine) or pure oxidizing (nitrogen tetroxide) propellants. Laboratory studies of this propellant inevitably show that for some test 
materials, it leaches minute amounts of metal ions. Nonetheless, safe, long-term storage of AF-M315E propellant in metallic and non-metallic tanks has been demonstrated ${ }^{8}$. For service components - valves, filters, elastomers, and lubricants - there is a growing set of materials where laboratory testing indicates sufficient compatibility for at least 3-5 year missions ${ }^{9}$.

A major effort of the GPIM program is to mature and qualify all AF-M315E propulsion system components for this mission, and for infusion on future space missions. As can be seen in the propulsion system schematic of Section III, AF-M315E-based advanced monopropellant systems are functionally equivalent to hydrazine systems, comprising the same number and type of components, but are distinct in that the different propellants have different material compatibilities. An extensive materials compatibility test campaign has been completed and confirms that all materials in system components that are wetted with the AF-M315E propellant are fully compatible. The thruster valve requires the most extensive modifications to ensure it is AF-M315E compatible. All wetted surfaces for the thruster valve, service valve, latch valve and propellant filter are being manufactured from materials which are fully compatible with this propellant.

Tests at elevated temperature revealed that the propellant tank elastomeric material met AMS-R-83412A specification requirements for compatibility. A longer term exposure test has been performed by AFRL, Edwards to determine any decrease in material functional properties and metal leaching profile over time. Previous compatibility studies have shown that the shell material of the selected tank has long-term compatibility with AF-M315E. compatibility testing of the bladder material has like-wise shown acceptable performance for multiyear missions, and hence made a wide variety of existing tanks applicable for the GPIM demonstration and future missions. This revelation is a major benefit for the infusion of the technology as it enables the use of simpler and lower cost elastomeric diaphragm tanks instead of more complex propellant management device (PMD) style tanks or metal diaphragm tanks. A study of the fracture behavior of the tank material with AF-M $315 \mathrm{E}$ is currently underway by NASA KSC in order to confirm that it complies with the fracture mechanics requirements of AFSPCMAN 91-710 for safe operation of a pressure vessel containing a non-hazardous fluid.

Table 2 summarizes component selections, respective mission readiness, and modifications required for the TDM green propulsion system. Existing hydrazine system components (TRL9, but evaluated at TRL6 for use with AF-M315E) comprise a nearly complete compatible set, with several components requiring straightforward modifications. The thruster valve will require the interior wetted surfaces to be lined with fully tested compatible material.

Table 2 Propulsion System Component Summary

\begin{tabular}{|l|c|c|c|}
\hline \multicolumn{1}{|c|}{ Component } & Design Adaptation & $\begin{array}{c}\text { TRL w/ } \\
\text { AF-M315E }\end{array}$ & TRL w/ Hydrazine \\
\hline Propellant Tank & No Change & 6 & 9 \\
\hline Thruster Valve & $\begin{array}{c}\text { Change wetted surface } \\
\text { material }\end{array}$ & 5 & 9 (similar $\mathrm{N}_{2} \mathrm{H}_{4}$ valve) \\
\hline Latch Valve & No Change & 6 & 9 \\
\hline System Transducer & No Change & N/A (gas side) & 9 \\
\hline Filter & No Change & 6 & 9 (similar $\mathrm{N}_{2} \mathrm{H}_{4}$ filter) \\
\hline Service Valves & $\begin{array}{c}\text { Change sealing ball } \\
\text { material }\end{array}$ & 5 & 9 (similar $\mathrm{N}_{2} \mathrm{H}_{4}$ valve) \\
\hline
\end{tabular}

\section{Propulsion System Payload Module Development Schedule}

The overall propulsion effort can principally be divided into two major efforts, development of the thrusters and manufacturing of the propulsion system,

Figure 12. Immediate system tasks included assessment of which system components to employ, and understanding of the scope of modifications needed to TRL9 hydrazine components for use with AF-M315E propellant. A complimentary effort was also initiated at the beginning to test the compatibility of all unknown materials with this green propellant. The flight system design effort has two phases, 1) system design up through PDR and 2) final system design up to CDR. The thruster development is divided into three phases: 1) Lab model $22 \mathrm{~N}$ thruster development, 2) Engineering model (EM) thruster design and then 3) the final flight design activity. Flight thruster designs are expected to be only minor modifications to the EM model based on lessons learned from the EM system bench testing. Testing is also principally divided into three tasks: 1) initial lab model testing of the $22 \mathrm{~N}$ thruster, 2) EM system bench level testing which includes assessment of both the $1 \mathrm{~N}$ and $22 \mathrm{~N}$ EM thruster designs as well as 
performance evaluation of the complete propulsion system with EM level components and 3) acceptance and qualification testing. Propulsion system delivery to Ball Aerospace Corporation is in April 2015.

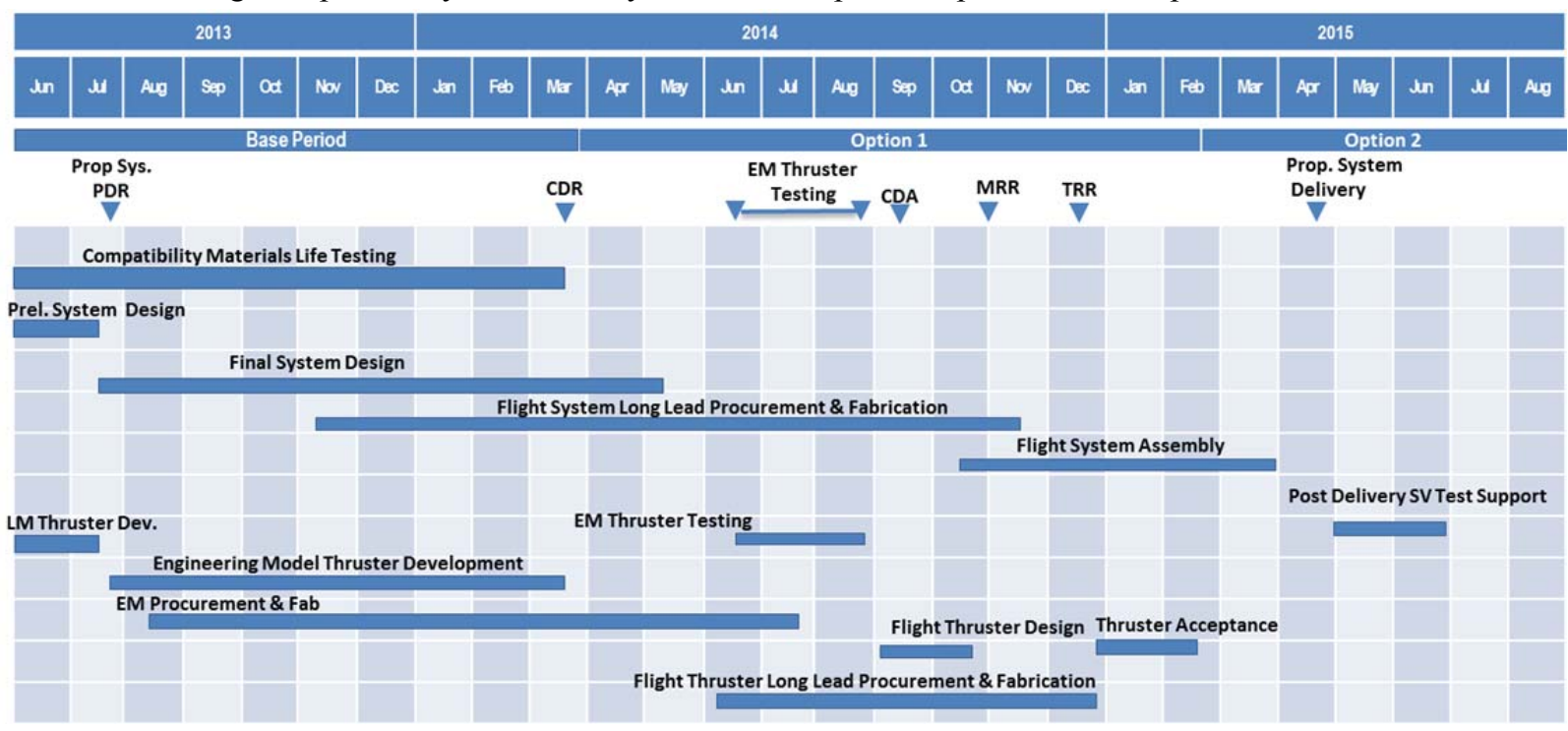

Figure 12 Propulsion System Schedule

\section{Conclusion}

The culmination of this program will be high-performance, green AF-M315E propulsion system technology at TRL 7+ that is ready for direct infusion to a wide range of applications for the space user community.

The combined benefits of low toxicity, easy open-container handling, and high performance of AF-M315E offer a strong alternative to hydrazine for dramatically reducing the cost of access to space for the small vehicles being developed by NASA, DoD and the commercial sector.

AF-M315E propulsion systems will enable spacecraft designers to accommodate significantly more propulsive performance than hydrazine, especially where volume is limited. Some differences in design considerations are needed over hydrazine systems, but in general the approaches are very similar. The GPIM demonstration program will show that these considerations are manageable, especially when compared to the significant benefits of AFM315E propulsion systems.

\section{Acknowledgments}

The authors wish to thank the NASA Space Technology Mission Directorate (STMD) in Washington DC and Technology Demonstration Mission (TDM) office out of NASA Marshall SFC for funding the GPIM technology demonstration program, NASA Glenn RC for their plume modeling and characterization efforts on the GPIM program and loan of data acquisition equipment, and AFRL Edwards AFB for their participation in the GPIM program in developing ground support equipment and loan of/assistance with the high precision thrust stand to be employed in testing of the GR-1 flight-weight engineering model thruster.

\section{References}

\footnotetext{
${ }^{1}$ McLean, C., Spores, R., Sheehy, J., "Green Propulsion Infusion Mission, Program Construct, and Mission Objectives", Commercial and Government Responsive Access to Space Technology Exchange (CRASTE) Conference, Bellevue, WA, 24-27 June, 2013.

${ }^{2}$ McLean, C., Spores, R., Sheehy, J., "Green Propulsion Infusion Mission, Program Construct, and Mission Objectives", $60^{\text {th }}$ JANAF Propulsion Meeting, Colorado Springs, CO, May 2013.
} 
${ }^{3}$ Mclean, C.H, Hale, M.J., Deininger, W. D., Spores, R.A., Frate, D.T., Johnson, W.L., Sheehy, J.A, “ Green Propellant Infusion Mission Program Overview”, 49th AIAA/ASME/SAE/ASEE Joint Propulsion Conference, San Jose, CA, July 2013.

${ }^{4}$ Deininger, W., et al, "Implementation of the Green Propellant Infusion Mission (GPIM) on a Ball Aerospace BCP-100 Spacecraft Bus", 49th AIAA/ASME/SAE/ASEE Joint Propulsion Conference, San Jose, CA, July 2013.

${ }^{5}$ Yim, J.T., Reed, B.D., Deans, M.C., McLean C.H., Sheehy, J.A., "Green Propellant Infusion Mission Plume Impingement Analysis", 49th AIAA/ASME/SAE/ASEE Joint Propulsion Conference, San Jose, CA, July 2013.

${ }^{6}$ Wucherer, E., Christofferson, S., Reed, B., "Assessment of High Performance HAN-monopropellants", AIAA-2000-3872, JPC, Huntsville, Al, July, 2000.

7 Meinhardt, D., Wucherer, E., Brewster, G., "HAN-Based Monopropellant Vehicle ACS Trade Study," Primex Aerospace Document R-97-2105, prepared for the NASA Lewis Research Center Launch Vehicle Project Office, September 1997.

${ }^{8}$ Wucherer, E., Morgan, R., Yoder, T.," Thermal Stability Testing of AF-M315E Propellant", 59thJPM/JANNAF, San Antonio, Tx, May 2012.

${ }^{9}$ Wucherer, E., Liu, J., ”AF-M315E Material Compatibility Study”, 59thJPM/JANNAF, San Antonio, Tx, May 2012. 